\title{
Concentration-Dependent Atypical Intestinal Absorption of Cyclic Phenylalanylserine: Small Intestine Acts as an Interface between the Body and Ingested Compounds
}

\author{
Takashi Mizuma, * Takuo Narasaka, and Shoji Awazu \\ Department of Biopharmaceutics and Drug Rationale Research Center, School of Pharmacy, Tokyo University of \\ Pharmacy and Life Science; 1432-1 Horinouchi, Hachioji, Tokyo 192-0392, Japan. \\ Received April 17, 2003; accepted July 16, 2003
}

\begin{abstract}
Intestinal absorption of peptides in linear form has been studied extensively, but there is little knowledge of peptides in a cyclic form. In this report, intestinal absorption of cyclic phenylalanylserine (cyclo(Phe-Ser)), a precursor of gliotoxin, was studied in isolated rat small intestine as a model cyclic dipeptide. Absorption clearance (CLabs) decreased in the presence of glycylsarcosine, cephalexin or cephradine, substrates for $\mathrm{H}^{+} /$oligopeptide cotransporter (PEPT1). CLabs of cyclo(Phe-Ser) also decreased at $4^{\circ} \mathrm{C}$, thus indicating that cyclo(Phe-Ser) is in part transported by PEPT1. However, the Eadie-Hofstee plot of absorption revealed an atypical profile at lower concentrations of cyclo(Phe-Ser) (around $0.1 \mathrm{~mm}$ ). Moreover, comparative experiments of absorptive and excretive transport showed that excretive transport from the serosal to mucosal side of isolated intestinal tissue at a $0.1 \mathrm{~mm}$ cyclo(Phe-Ser) was superior to absorptive transport from the mucosal side to the serosal side, and vice versa at a $1 \mathrm{~mm}$ cyclo(Phe-Ser). A kinetic model was constructed, in which cyclo(Phe-Ser) concentration for excretive transport was assumed to be at the binding site of excretive transporter, but not the unbound cytoplasmic concentration. These results as well as the results of kinetic analysis indicate that intestinal absorption consists of passive transport, carrier-mediated absorptive transport by PEPT1 and carrier-mediated excretive transport, resulting in atypical absorption. Although cyclic dipeptides have potentials as drugs, their intestinal absorption may be complex. The results of this study lead us to conclude that absorptive and excretive transport by the small intestine acts as an interface between the body and ingested compounds.
\end{abstract}

Key words cyclic phenylalanylserine; cyclic dipeptide; $\mathrm{H}^{+}$/oligopeptide cotransporter; atypical absorption; excretive transport

Recently it has been reported that cyclic dipeptides have various biological activities such as anti-tumor and antibiotic activities. $^{1,2)}$ Although the intestinal absorption of linear dipeptides has been studied extensively, ${ }^{3)}$ the intestinal absorption of cyclic dipeptides has not yet been characterized in detail. To develop these valuable cyclic dipeptides as orally active drugs requires clarifying the mechanism of their intestinal absorption. We found that cyclic glycylphenylalanine (cyclo(Gly-Phe)) and cyclic seryltyrosine (cyclo(SerTyr)) are stable compounds and are transported by intestinal $\mathrm{H}^{+}$/oligopeptide cotransporter (PEPT1). ${ }^{4,5}$ These cyclic dipeptides have an amide bond and an aromatic ring, and are similar in structure to cephalexin and cephadroxil, which are known to be transported by PEPT1.,6) Furthermore, cyclic dipeptides are structurally rigid and chemically stable in the intestine. ${ }^{4,5,7)}$ Cyclic phenylalanylserine (cyclo(Phe-Ser)), which is an intermediate in the biosynthesis of gliotoxin, ${ }^{8)}$ is similar in structure to cyclo(Gly-Phe) and cyclo(Ser-Tyr). In this study, therefore, to fundamentally characterize cyclic dipeptides absorption based on structural similarity, the intestinal transport of cyclic phenylalanylserine (cyclo(PheSer)) was studied.

\section{MATERIALS AND METHODS}

Chemicals Cyclo(Phe-Ser) was purchased from Bachem Feinchemikalien AG (Switzerland). Glycylsarcosine, cephalexin and cephradine were purchased from Sigma (St. Louis, MO, U.S.A.).

Absorption Experiments Experiments on absorption of cyclo(Phe-Ser) were performed as previously reported. ${ }^{4,5}$
Briefly, after overnight fast, male Wistar rats $(180-220 \mathrm{~g}$, Japan Slc Inc., Shizuoka, Japan) were anesthetized with ether. The small intestine was isolated and was everted in cold saline on ice. The intestine $(10 \mathrm{~cm})$ was cannulated with glass canulae at each end and then connected to a disposable $10 \mathrm{ml}$ plastic syringe. The everted intestine was placed in $30 \mathrm{ml}$ of incubation medium (modified Krebs-Ringer-bicarbonate phosphate buffer, $\mathrm{pH}$ 7.4) containing cyclo(Phe-Ser) in a beaker through which gas $\left(95 \% \mathrm{O}_{2}, 5 \% \mathrm{CO}_{2}\right)$ was bubbled. The serosal side was filled with $5 \mathrm{ml}$ of incubation medium without cyclo(Phe-Ser). The medium $(0.1 \mathrm{ml})$ was periodically sampled from both the serosal and mucosal sides over a 40-minute period. One hundred microliters of $10 \%$ perchloric acid containing $1 \mathrm{~mm} p$-hydroxybenzoic acid as an internal standard for HPLC was mixed with a $100 \mu 1$ sample in a plastic microtube. After centrifugation at $11000 \times \boldsymbol{g}$ for 5 min, the resulting supernatant was assayed by HPLC.

Comparative Experiments of Absorptive and Excretive Transport Four $\mathrm{cm}$ of small intestine located $20 \mathrm{~cm}$ below the Treitz ligament was isolated from rat. The intestinal tissue was stripped of its muscular layer and then mounted in the diffusion chamber $\left(0.64 \mathrm{~cm}^{2}\right.$, Costar, Japan $)$. To examine absorptive transport from the mucosal (donor) to serosal (reservoir) side, $1.5 \mathrm{ml}$ of cyclo(Phe-Ser)-containing incubation buffer was added to the mucosal side, and $1.5 \mathrm{ml}$ of incubation buffer alone was added to the serosal side. For excretive transport, from the serosal (donor) to mucosal (reservoir) side, the experiment was carried out in reverse. Gas $\left(95 \% \mathrm{O}_{2}, 5 \% \mathrm{CO}_{2}\right)$ was supplied to incubation buffer on both the serosal and mucosal sides, and transport experiments were performed for $60 \mathrm{~min}$ at $37^{\circ} \mathrm{C}$. The incubation medium 

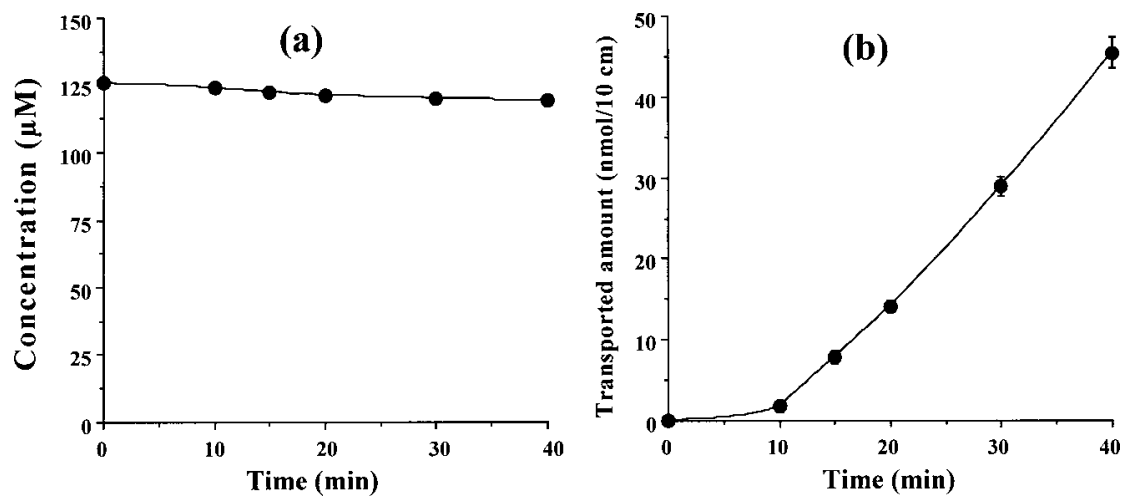

Fig. 1. Time Course of Cyclo(Phe-Ser) on Mucosal (a) and Serosal (b) Sides in Intestinal Absorption

Cyclo(Phe-Ser) concentration: $125 \mu$ m. Data represent means \pm S.E. $(n=4-15)$

$(0.1 \mathrm{ml})$ was periodically sampled from the reservoir side and $0.1 \mathrm{ml}$ of fresh incubation buffer was supplemented. Sample was mixed with $0.1 \mathrm{ml}$ of $10 \%$ perchloric acid containing $1 \mathrm{~mm} p$-hydroxybenzoic acid as an internal standard for HPLC assay in a plastic microtube. After centrifugation at $11000 \times \boldsymbol{g}$ for $5 \mathrm{~min}$ the resulting supernatant was assayed by HPLC.

HPLC Assay Cyclo(Phe-Ser) was determined by HPLC. The HPLC system (Hitachi, Tokyo, Japan) consisted of a 655A-11 pump, a 655A UV detector (194 nm) with a D-2500 integrator, and an ODS-120T column $(4.6 \mathrm{~mm}$ i.d., $250 \mathrm{~mm}$ length, Tosoh Corp., Tokyo). The mobile phase consisted of $15 \%$ methanol and $0.05 \%$ phosphoric acid in water and had a flow rate of $1.5 \mathrm{ml} / \mathrm{min}$.

Data Analysis Absorption clearance (CLabs) was calculated by Eq. 1 .

$$
\text { CLabs }=\frac{\text { absorption rate }}{\text { concentration on the mucosal side }}
$$

Kinetic analysis was performed by fitting data to Eq. 3 with the nonlinear fitting program MULTI. ${ }^{9)}$

$$
\begin{aligned}
\text { absorption rate } & =\frac{\text { Vmax, abs } \times C}{\mathrm{Km}, \mathrm{abs}+\mathrm{C}}-\frac{\mathrm{Vmax}, \mathrm{exc} \times \mathrm{Ccel}}{\mathrm{Km}, \mathrm{exc}+\mathrm{Ccel}}+\mathrm{CLpas} \times \mathrm{C} \\
& =\frac{\mathrm{Vmax}, \mathrm{abs} \times \mathrm{C}}{\mathrm{Km}, \mathrm{abs}+\mathrm{C}}-\frac{\mathrm{Vmax}, \mathrm{exc} \times \mathrm{C}}{\mathrm{Km}, \mathrm{exc} / \mathrm{Kp}+\mathrm{C}}+\mathrm{CLpas} \times \mathrm{C} \\
\mathrm{Kp}=\frac{\mathrm{Ccel}}{\mathrm{C}} &
\end{aligned}
$$

Vmax,abs and Vmax,exc represent maximum rate for absorptive transport and excretive transport, respectively. $\mathrm{Km}$, abs and $\mathrm{Km}$,exc represent Michaelis constant for absorptive transport and excretive transport, respectively. CLpas is transport clearance by passive diffusion. $\mathrm{C}$ and Ccel represent concentrations of cyclo(Phe-Ser) on the mucosal side and at the binding site of the excretive transporter, respectively.

Statistical analysis was performed by Dunnett multiple comparison post tests following analysis of variance (ANOVA).

\section{RESULTS}

Time Course of Intestinal Absorption of Cyclo(PheSer) Figure 1 shows the time course of intestinal absorp- tion of cyclo(Phe-Ser). Cyclo(Phe-Ser) is stable on the mucosal side (Fig. 1a) and can be transported to the serosal side (Fig. 1b). The absorption rate became constant after $15 \mathrm{~min}$.

Effect of PEPT1 Substrate on Absorption of Cyclo(Phe-Ser) CLabs of cyclo(Phe-Ser) under various conditions is shown in Fig. 2. CLabs of cyclo(Phe-Ser) decreases in the presence of $10 \mathrm{~mm}$ glycylsarcosine, cephalexin

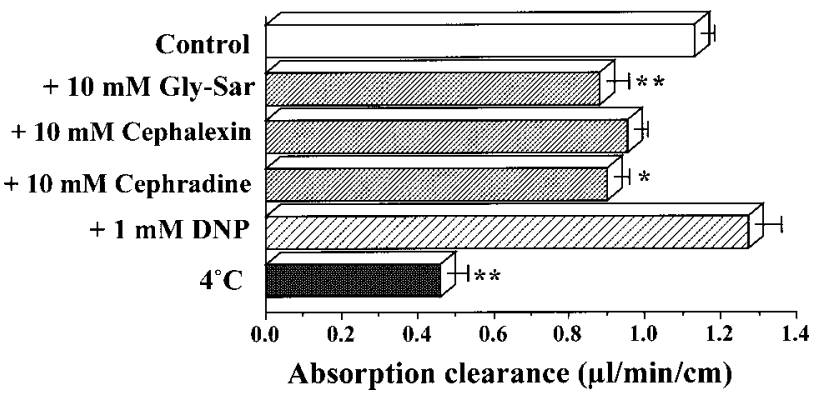

Fig. 2. Effects of PEPT1 Substrate on Absorption of Cyclo(Phe-Ser) in Small Intestine

Cyclo(Phe-Ser) concentration: $125 \mu \mathrm{M}$. Data represent means \pm S.E. $(n=3-15)$. $* p<0.05, * * p<0.01$ versus control.

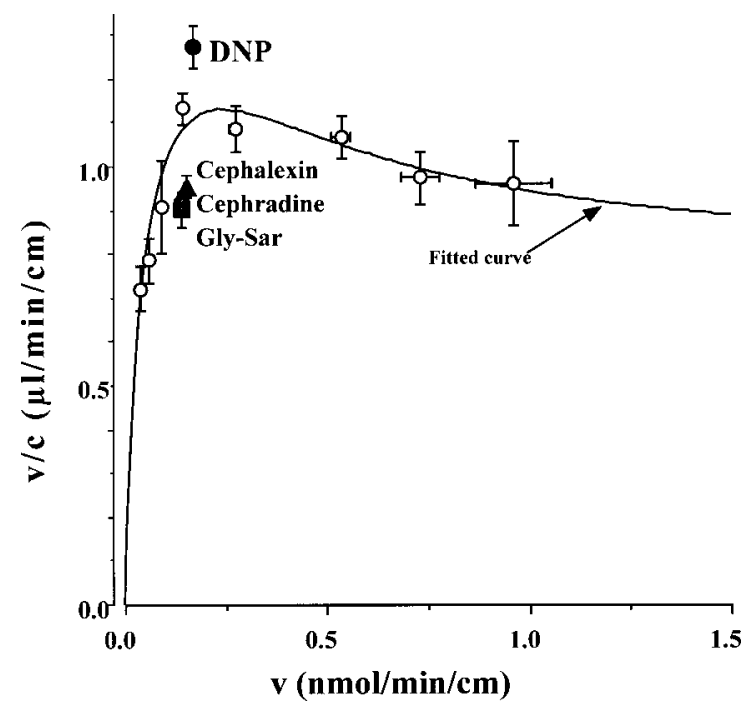

Fig. 3. Eadie-Hofstee Plot of Cyclo(Phe-Ser) Absorption in Small Intestine

Data represent means \pm S.E. $(n=3-15)$. $\mathbf{\square}$, plus $10 \mathrm{~mm}$ glycylsarcosine; $\boldsymbol{\nabla}$, plus $10 \mathrm{~mm}$ cephradine; $\boldsymbol{\Delta}$, plus $10 \mathrm{~mm}$ cephalexin; $\boldsymbol{\bullet}$, plus $1 \mathrm{~mm} \mathrm{DNP;}-$, fitted curve based on Eq. 3 (see text). Cyclo(Phe-Ser) concentration: 0.075 to $1 \mathrm{~mm}$. 

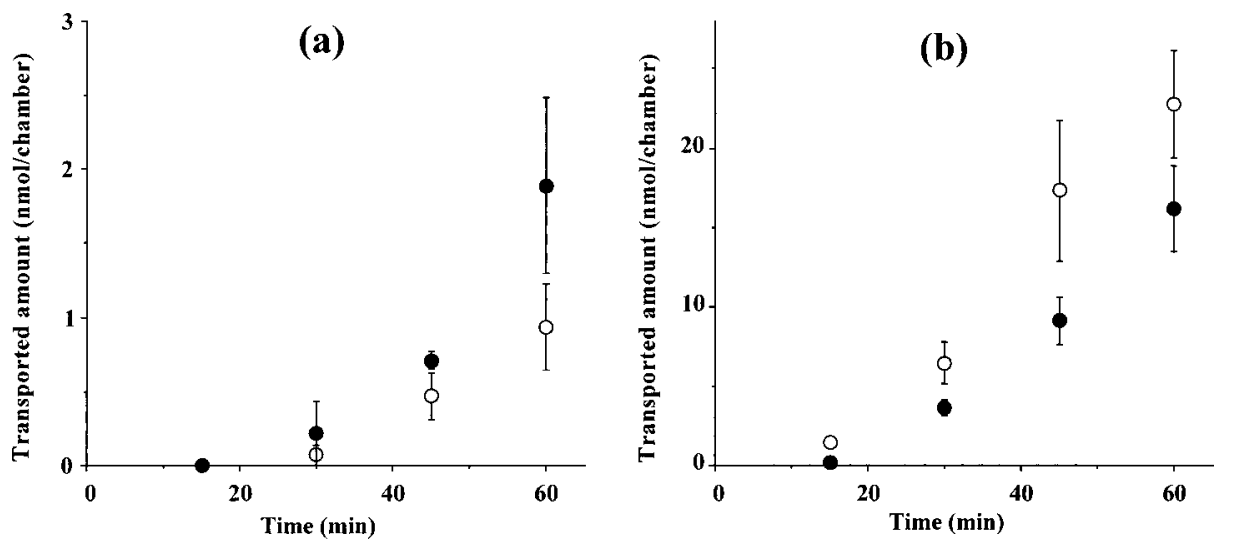

Fig. 4. Comparison of Absorptive and Excretive Transport of Cyclo(Phe-Ser) at 0.1 (a) and $1 \mathrm{~mm}$ (b) across Small Intestine Data represent means \pm S.E. $(n=6)$. $\bigcirc$, from the mucosal to serosal sides; $\bullet$, from the serosal to mucosal sides.

or cephradine, all PEPT1 substrates. CLabs of cyclo(PheSer) also decreases at $4{ }^{\circ} \mathrm{C}$, and there was a tendency for CLabs of cyclo(Phe-Ser) to increase in the presence of $1 \mathrm{~mm}$ DNP, though not significant.

Concentration Dependency of CLabs Eadie-Hofstee plot shows an atypical profile for cyclo(Phe-Ser) absorption (Fig. 3). Although CLabs of cyclo(Phe-Ser) increases with increases in concentration up to $0.2 \mathrm{~mm}$, it decreases with increases in concentration over $0.2 \mathrm{~mm}$. The kinetic parameters were Vmax,abs $=0.367 \mathrm{nmol} / \mathrm{min} / \mathrm{cm}, \mathrm{Km}, \mathrm{abs}=222 \mu \mathrm{M}$ for absorptive transport; Vmax,exc $=0.117 \mathrm{nmol} / \mathrm{min} / \mathrm{cm}$, $\mathrm{Km}, \mathrm{exc} / \mathrm{Kp}=31.1 \mu \mathrm{M}$ for excretive transport; CLpas= $0.762 \mu \mathrm{l} / \mathrm{min} / \mathrm{cm}$ for passive transport.

The data is well represented by the fitted curve based on Eq. 3, which consists of 3 terms: carrier-mediated absorptive transport, carrier-mediated excretive transport and passive transport. In the presence of PEPT1 substrates, CLabs at cyclo(Phe-Ser) concentration of $0.125 \mathrm{~mm}$ was lower than that of the fitted curve, whereas CLabs of cyclo(Phe-Ser) at $0.125 \mathrm{~mm}$ in the presence of DNP was higher than that of the fitted curve.

Comparison of Absorptive Transport and Excretive Transport Figures $4 \mathrm{a}$ and $\mathrm{b}$ show absorptive transport from the mucosal to serosal side and excretive transport from the serosal to mucosal side at 0.1 and $1 \mathrm{~mm}$ cyclo(Phe-Ser). The absorptive transport of cyclo(Phe-Ser) from the mucosal to serosal side is higher than the excretive transport from the serosal to mucosal side at $1 \mathrm{~mm}$, while the excretive transport of cyclo(Phe-Ser) from the serosal to mucosal side is higher than the absorptive transport from the mucosal to serosal side at $0.1 \mathrm{~mm}$.

\section{DISCUSSION}

PEPT1-Mediated Transport Since the absorption rate became constant $15 \mathrm{~min}$ after initiating the experiment (Fig. 1), CLabs of cyclo(Phe-Ser) was calculated according to Eq. 1 from data obtained in minutes 15 through 30. CLabs of cyclo(Phe-Ser) decreased in the presence of PEPT1 substrates such as glycylsarcosine, cephalexin and cephradine ${ }^{3,6)}$ and in response to lowering temperature to $4{ }^{\circ} \mathrm{C}$ (Fig. 2). These results indicate that cyclo(Phe-Ser) is transported in part by PEPT1. Also, it appears that another transport carrier may be involved in the cyclo(Phe-Ser) absorption, because there was a difference in CLabs of cyclo(Phe-Ser) in the presence of PEPT1 substrate and at $4{ }^{\circ} \mathrm{C}$.

Atypical Absorption and Asymmetrical Transport The Eadie-Hofstee plot of cyclo(Phe-Ser) absorption was atypical (Fig. 3). CLabs of cyclo(Phe-Ser) decreased with increases in concentration above $0.2 \mathrm{~mm}$, indicating typical carrier-mediated absorption. However, CLabs of cyclo(Phe-Ser) increased in concentration up to $0.2 \mathrm{~mm}$, suggesting that carrier-mediated excretive (anti-absorptive) transport is also involved in cyclo(Phe-Ser) transport. Therefore, absorptive transport of $0.1 \mathrm{~mm}$ and $1 \mathrm{~mm}$ cyclo(Phe-Ser) from the mucosal to serosal side was compared with excretive transport of $0.1 \mathrm{~mm}$ and $1 \mathrm{~mm}$ cyclo(Phe-Ser) from the serosal to mucosal side (Fig. 4). Absorptive transport rate was not equal to excretive transport rate. The transport of cyclo(Phe-Ser) from the mucosal to serosal side was higher than that from the serosal to mucosal side at $1 \mathrm{~mm}$ cyclo(Phe-Ser), indicating absorptive transport is superior to excretive transport. In contrast, the transport of cyclo(Phe-Ser) from the serosal to mucosal side was higher than that from the mucosal to serosal side at $0.1 \mathrm{~mm}$ cyclo(Phe-Ser), indicating that excretive transport is superior to absorptive transport.

Effects of PEPT1 Substrate and DNP on Atypical Absorption Kinetics of Cyclo(Phe-Ser) Since the transport of cyclo(Phe-Ser) across the intestinal membrane was asymmetrical, the data was input into Eq. 3 (Fig. 3). The fitted curve matched the absorption data well, indicating that intestinal absorption of cyclo(Phe-Ser) consists of the three components contained within that equation: carrier-mediated absorptive transport, carrier-mediated excretive transport and passive transport. CLabs of $0.125 \mathrm{~mm}$ cyclo(Phe-Ser) in the presence of PEPT1 substrate was lower than the fitted curve, indicating that cyclo(Phe-Ser) is transported in part by PEPT1. On the other hand, CLabs of $0.125 \mathrm{~mm}$ cyclo(PheSer) in the presence of DNP was higher than the fitted curve. Since DNP inhibits ATP production, the excretive transport of cyclo(Phe-Ser) is believed to be mediated by a transport carrier that is more closely linked to ATP than PEPT1, and that thereby DNP increased CLabs of $0.125 \mathrm{~mm}$ cyclo(PheSer).

Kinetic Aspects of Excretive Transport Significant influence of excretive transport by way of P-glycoprotein on intestinal absorption has been reported (for example, see ref. 10). However, no kinetic model for excretive transport in- 
volved in the drug absorption has been established. Recently, Rosenberg et al. reported that drugs (transport substrate) directly access from the lipid bilayer of plasma membrane to the central pore of the transporter. ${ }^{11)}$ This indicates that it is important to define the concentration (Cexc) of substrate which exists at the binding site of the transporter in the lipid bilayer. Lentz et al. defined the substrate concentration relevant to the binding site of excretive transporter as the unbound cytoplasmic drug concentration. ${ }^{12)}$ At present, there is no consensus on the substrate concentration, and it is crucial to find what concentration is valid for the excretive transporter in drug absorption kinetics. Furthermore, the mechanism for the excretive transport of cyclo(Phe-Ser) is unclear; we therefore proposed Eq. 3. Our next target is to find how $\mathrm{Kp}$ is represented and what the transporter is.

In conclusion, not only absorptive transport, but also excretive transport of cyclo(Phe-Ser) was observed. That is, the intestinal absorption of cyclo(Phe-Ser) consists of carriermediated absorptive transport and carrier-mediated excretive transport in addition to passive transport. Absorptive transport is mediated by PEPT1, whereas excretive transport is mediated by a transporter that is closely linked to ATP. The concentration-dependent preference of these absorptive and excretive transport cause atypical intestinal absorption of cyclo(Phe-Ser). The small intestine acts as an interface between the body and ingested compounds by absorptive and excretive transport.
Acknowledgement The authors thank Ms. Wakako Hiyoshi for technical assistance.

\section{REFERENCES}

1) Milne P. J., Hunt A. L., Rostoll K., Van Der Walt J. J., Graz C. J. M., J. Pharm. Pharmacol., 50, 1331-1337 (1998).

2) Graz M., Hunt A., Jamie H., Grant G., Milne P., Pharmazie, 54, 772775 (1999).

3) Fei Y.-J., Kanai Y., Nussberger S., Ganapathy V., Leibach F. H., Romero M. F., Singh S. K., Boron W. F., Hediger M. A., Nature (London), 368, 563-566 (1994).

4) Mizuma T., Masubuchi S., Awazu S., J. Pharm. Pharmacol., 49, 1067-1071 (1997).

5) Mizuma T., Masubuchi S., Awazu S., J. Pharm. Pharmacol., 50, 167172 (1998).

6) Wenzel U., Gebert I., Weintraut H., Weber W.-M., Claub W., Daniel H., J. Pharmacol. Exp. Pharmacol., 277, 831-839 (1996).

7) Mizuma T., Koyanagi A., Awazu S., Biochim. Biophys. Acta, 1475, 90-98 (2000).

8) Kirby G. W., Patrick G. L., Robins D. J., J. Chem. Soc. Perkin Trans. I, 1978, 1336-1338.

9) Yamaoka K., Tanigawara Y., Nakagawa T., Uno T., J. PharmacobioDyn., 4, 879-885 (1981).

10) Tsuji A., Tamai I., Pharm. Res., 13, $963-977$ (1996).

11) Rosenberg M. F., Kamis A. B., Callaghan R., Higgins C. F., Ford R. C., J. Biol. Chem., 278, 8294-8299 (2003).

12) Lentz K. A., Polli J. W., Wring S. A., Humphreys J. E., Polli J. E., Pharm. Res., 17, 1456-1460 (2000). 